Restoring the Blessings of the Morning Star: Childbirth and Maternal-Infant Health for First Nations near Edmonton, Alberta

Adrienne Dawn Wiebe, Mennonite Central Committee

Sylvia Barton, Associate Professor, Faculty of Nursing, University of Alberta

Laura Auger, Aboriginal Health Coordinator, Sturgeon Community Hospital, Alberta Health Services

Em Pijl-Zieber, Instructor, Faculty of Health Sciences, University of Lethbridge

Caroline Foster-Boucher, Instructor and Aboriginal Nursing Coordinator, Faculty of Nursing, University of Alberta

aboriginal policy studies Vol. 5, no. 1, 2015, pp. 47-68

This article can be found at:

http://ejournals.library.ualberta.ca/index.php/aps/article/view/23823

ISSN: $1923-3299$

Article DOI: http://dx.doi.org/10.5663/aps.v5i1.23823

aboriginal policy studies is an online, peer-reviewed and multidisciplinary journal that publishes original, scholarly, and policy-relevant research on issues relevant to Métis, non-status Indians and urban Aboriginal people in Canada. For more information, please contact us at apsjournal@ualberta.ca or visit our website at www.nativestudies.ualberta.ca/research/aboriginal-policy-studies-aps. 


\title{
Restoring the Blessings of the Morning Star: Childbirth and Maternal-Infant Health for First Nations near Edmonton, Alberta $^{1}$
}

\author{
Adrienne Dawn Wiebe \\ Mennonite Central Committee \\ Sylvia Barton \\ Associate Professor, Faculty of Nursing, University of Alberta \\ Laura Auger \\ Aboriginal Health Coordinator, Sturgeon Community Hospital, Alberta Health Services \\ Em Pijl-Zieber \\ Instructor, Faculty of Health Sciences, University of Lethbridge \\ Caroline Foster-Boucher \\ Instructor and Aboriginal Nursing Coordinator, Faculty of Nursing, University of Alberta
}

\begin{abstract}
It is not only remote Aboriginal communities in Canada that have poorer maternalinfant health status than Canadian averages; residents of First Nation communities located close to large urban centres also experience this health status gap. Alexander, Alexis, Enoch, and Paul First Nations are located within an hour of healthcare services in greater Edmonton. The narratives of 75 predominantly Cree and Stoney women from these communities were gathered through seven talking circles and five semi-structured interviews. The participants described their experiences of loss and separation as pregnancy care and childbirth moved out of the community and into the hospital over the last two generations. This shift was not only a geographic relocation; it also disconnected the childbirth experience from elders, family and community, traditional teachings, and spiritual meaning. Conversely, the participants' hospital experiences were characterized by a limited sense of cultural safety. Participants highlighted the urgent need to reintegrate culturally based community support and health perspectives into the childbirth experience. The implementation of such a culturally integrated healthcare model in all Aboriginal communities - remote, rural, suburban, and urban-may be the key finally to closing the gap between Aboriginal and non-Aboriginal maternal and infant health status in Canada.
\end{abstract}

1 Acknowledgements: The authors would like to express their deep gratitude to all the participants who generously shared their experiences and insights in this study. We especially appreciate and learned from the wisdom of the following Elders: Rudy Bird, Martha Campiou, Pearl Gordon, Violet Nelson, and Lillian Shirt. In addition, the authors greatly appreciate the thoughtful and thorough feedback that the reviewers provided, which greatly strengthened this paper. We would also like to thank the Alberta Centre for Child, Family \& Community Research for providing a research grant for this study.

aboriginal policy studies, vol. 5, no. 1, 2015

www.nativestudies.ualberta.ca/research/aboriginal-policy-studies-aps

ISSN: $1923-3299$ 


\section{Introduction}

"The Morning Star is where we get our matriarchal blessings ... One of the blessings we get from the Creator is the blessing of the womb; [the Creator] blessed us to bring life into this world."

- Elder Participant

This study explores the childbirth experiences of several generations of women from First Nations communities located relatively close to a large Canadian city. The impact of the institutionalization and medicalization of childbirth on the health of Aboriginal mothers and newborns compared to that of the general population has been widely documented in Canada (Allan and Smylie 2015; Smylie et al. 2010; Lalonde et al. 2009). The removal of childbirth from the family and cultural context to city hospitals has not had the positive results expected with the provision of modern Western medical services. While there has been considerable research to date on this topic in remote rural locations in Canada, the experience of women from communities close to large urban centres has not been the subject of many studies of this kind (Wright 2015; SOGC 2010).

In the present study, several generations of Aboriginal women and elders from four non-remote Cree and Stoney communities-Alexander, Alexis, Enoch, and Paul First Nations-located within seventy-five kilometers of Edmonton, Alberta, share their stories of childbirth from the 1950s to the present. The participants identify the gaps and issues that currently exist between modern Western medicine and Indigenous traditional approaches to childbirth, and how these might be harmonized to promote better health outcomes for mothers and babies, as well as the family and community at large. The findings will be of interest to Western healthcare providers, traditional health practitioners, and community leaders looking for ways to improve the health of their communities and to restore the "blessings of the Morning Star."

\section{Review of the Relevant Literature}

Disparities in maternal-infant health between Aboriginal and non-Aboriginal peoples in Canada persist despite universal healthcare access and some narrowing of the gap in health indicators in the past three decades (Allan and Smylie 2015; Olsen et al. 2012; Smylie et al. 2010; Adelson 2005). Significantly, distance to healthcare services, and therefore access to services, does not appear to be the only factor in this health gap. There is evidence that Aboriginal maternal-infant health status does not improve with proximity to urban centres in the way that non-Aboriginal health status does (Lawford and Giles 2012; Lou et al. 2010). For Aboriginal mothers who live in rural communities close to large urban centers, access is still a problem, particularly in circumstances requiring complex obstetric care (Claydon et al. 2007). This move has been largely policy-driven, as well as part of general societal trends in the last sixty years to medicalize and institutionalize childbirth. 
In addition to access, the other key determinant of health that continues to frustrate efforts to improve maternal-infant health for Aboriginal communities close to cities appears to be the model of healthcare itself. The critical importance of culturally appropriate and community-based health approaches for ethnic and culturally diverse minorities has been documented, particularly in relation to Aboriginal mental and physical health in Canada (Martin Hill 2009; Reading et al. 2007). There is a growing body of evidence for the association among cultural continuity, integration and resiliency, as well as selfdetermination and political empowerment, with healing and well-being in Aboriginal communities (Kirmayer and Valaskakis 2009; Greenwood 2005).

Positive health outcomes and resiliency are also dependent on family, community, cultural and spiritual continuity factors (Allan and Smylie 2015; Lawford and Giles 2012; SOGC 2010; Chandler and Lalonde 2008). As Lalonde, Butt and Bucio suggest, "without addressing the cultural, and possibly linguistic, barriers that impede Aboriginal people in seeking the services they need, the proximity of these services is irrelevant" $(2009,957)$.

Colonization and assimilation had a profoundly negative impact on the health of Aboriginal peoples and communities in Canada (Allan and Smylie 2015; Moffit 2004). The systematic suppression of traditional beliefs, customs and practices contributed to a concomitant erosion of health that is still present today. Compounding the elimination of culturally-based health supports was the removal of health services from the communities to non-Aboriginal, federal entities (Lalonde et al. 2009). In removing childbirth from Aboriginal communities, not only was access to health care limited or denied, but the accumulated wisdom and traditions of elders and midwives was also lost.

As Wright describes, the policy of evacuation was developed over twenty years starting in the 1960s because it was "believed that the hospitals with advanced equipment and services available were the safest in which to birth infants" $(2015,3)$. Women were moved to tertiary care facilities in urban centres in southern Canada, often weeks before their due dates, to await childbirth. This policy largely applied to remote communities in northern Canada; however, Aboriginal communities situated closer to urban centres also felt its impact: while women in the latter category were not removed from their communities before their due dates, they were expected to deliver in hospitals (Lawford and Giles 2012; SOGC 2010).

In the last decade, a determinants-of-health approach has been used in research with Aboriginal communities in Canada to identify the elements of best practice in the provision of health services for Aboriginal peoples. The "Maternal/Infant Health in Canada" report from the National Aboriginal Health Organization, for example, recommends 1) the development of health education, promotion, and services that address a range of identified issues and inequities reflecting First Nations, Inuit, and Métis values, traditions, cultures, and languages; 2) broadening the participation of Aboriginal women and youth in health research, policy planning, and decision-making; and 3) obtaining knowledge by gathering sound data about the health of First Nations, Inuit, and Métis women and girls (NAHO 2004). 
A recent review of the literature on best practices in culturally competent care for Aboriginal women in hospital settings highlighted the importance of Aboriginal health perspectives, beliefs and practices (Birch et al. 2009). The researchers emphasized the importance of such overarching elements of culturally competent care as the application of knowledge, self-awareness, skills, personal attributes, attitudes, and respectful partnerships with communities. Such key concepts must be embraced by healthcare providers in the provision of appropriate care and services in relation to clients' cultural characteristics. In addition, there is a need for more community-specific research that identifies particular inequities related to geography, culture, and personal living conditions.

The importance of responsiveness to culture and community specificity was prominent in a review of midwifery and birthing services in remote Canadian Aboriginal communities (Couchie and Sanderson 2007). The study identified the need for healthcare providers to be informed about 1) the health inequities of Aboriginal peoples in relation to broader determinants of health; 2) the importance of communities and healthcare institutions learning to work together effectively; and 3) the integral part played by midwifery in maternity care in Aboriginal communities. In a more localized context, a review of research on Mi'kmaq women's childbirth experiences in hospital settings indicates that 1) the complexities of cultural differences were often not understood by mainstream healthcare providers; 2) these birth experiences had profound implications for healthcare outcomes; and 3) only limited research specific to the Mi'kmaq had been conducted (Whitty-Rogers 2006).

At the same time as the mainstream healthcare system has begun to recognize the cultural and racial nature of health disparities in Canada over the last two decades, there has been concurrent revitalization of traditional Aboriginal midwifery in Canada. As a result of this, three Aboriginal birthing centres have been established that harmonize traditional knowledge, medicine, and healing practices with the Western health system (Skye 2010). Two of these birthing centres are in remote locations in Nunavut and northern Quebec, and the third one is on the Six Nations First Nation, within an hour's drive of the city of Hamilton in southern Ontario. Additionally, midwifery training and provision of services have been established in many areas across Canada (Lalonde et al. 2009, 959-60).

More recently, the original model of a culturally-responsive healthcare service that "integrates" Western and Aboriginal health systems has been further developed with the concept of a "harmonized" system of healthcare. While "integration" suggests the merging of two systems, "harmonization" aims to enable each system to maintain its integrity as a holistic model while working in coordination with the other to meet the needs of those requiring healthcare services (PAHO/WHO 2011). This approach has important potential for the Canadian context, particularly in relation to the future of Aboriginal maternalinfant health.

While the basic elements of a culturally safe healthcare system that harmonizes allopathic and Indigenous health practices and pays attention to power relations between service providers and service users have been identified, the needs of each region are 
shaped by particular geographic, historical, cultural, and community characteristics (Allan and Smylie 2015; Lang et al. 2010; Foster 2006). There is not a one-size-fits-all prescription that can be implemented across Canada to harmonize Aboriginal perspectives and cultural requirements with mainstream healthcare services. Each cultural region and community needs to develop its own programs based in the local context of culture, whereby relevant and culture-specific services may be offered to women, children, families and communities. The present study contributes to the understanding of how to do this with the communities around Edmonton.

\section{Methods}

The authors recognize the inherent dangers of generalizing and stereotyping cultural groups, potentially negating the myriad of differences that exist among Aboriginal cultures and nations. While there may be patterns and similarities among Aboriginal cultures, any generalization should be considerate and respectful of the important differences among peoples and cultures. In this paper, the term "Aboriginal" is used to denote all peoples of First Nations, Inuit and Métis heritage; specific First Nations and communities are identified by name.

\section{Study Design}

This study used as guidelines the principles of community-based, participatory research (Minkler and Wallerstein 2008) in its endeavor. Aboriginal community members guided our involvement and contributed to a better understanding of Aboriginal women's past and present experiences of childbirth, social issues relevant to maternal and infant health, and perceived quality of family life in and around Edmonton, Alberta. Sensitivity to each of these dimensions positioned the research team to be better able to elicit the cultural findings of the study; and to understand revelations about how Aboriginal knowledge and experience can provide insights into the development of culturally safe Aboriginal programs that are integrated with, or more autonomous from, existing services.

A descriptive qualitative study (Sandelowski 2000) was the method of choice, in which straight descriptions of phenomena were used to determine the who, what, and where of events. This methodology resulted in 1) an understanding of Aboriginal maternal and infant health history and practices within the study communities, 2) the gathering of women's narratives of their childbirth experiences in the healthcare system in the Greater Edmonton area, and 3) the identification of recommendations to improve the experiences and health outcomes of Aboriginal women and infants.

\section{Participants}

The First Nations people in the Edmonton region are largely of the Cree, Stoney, and Nakota-Sioux Nations, with smaller numbers of Salteaux and Dene. This study involved four First Nations communities and three urban Aboriginal organizations in and near 
Edmonton. Over a period of six months, seventy-five predominantly Cree and Stoney women participated in seven talking circles. These communities are relatively close to urban healthcare services such as the Westview Hospital and Health Centre in Stony Plain, several hospitals with obstetrics units, and the tertiary healthcare facility of the Royal Alexandra Hospital in the centre of Edmonton, which provides specialized obstetric care.

Four talking circles were held on the four First Nations around Edmonton: Alexander, Alexis, Enoch, and Paul First Nations. Talking circles were also held at Bent Arrow Traditional Healing Society in Edmonton, and at the Aboriginal Family Resource Centre in Stony Plain, forty kilometres from Edmonton, which is also the location of a secondary hospital. Additionally, four Cree and Stoney traditional elders and one younger woman participated in individual interviews.

The sample size was obtained by purposeful sampling to ensure comprehensive representation. Participants were recruited through healthcare and service providers that serve the four First Nations communities, as well as several Aboriginal community organizations that had a trusting rapport with members of the research team, particularly the Cree research team member. Individuals who wanted to contribute their experience and input to the study regarding Aboriginal women's maternal and infant health needs in the region were recruited.

\section{Ethics/Consent}

Approaches to research involving Aboriginal Peoples (Canadian Institutes of Health Research 2007) that build on ethical policies for research involving Aboriginal Peoples (Tri-council Policy Statement 2, 2010) served as guidelines for this research. The study was approved by the Health Research Ethics Board at the University of Alberta. Information letters and informed consent forms in English were given to participants and signed by all participants. The information was translated by the Cree research team member for one Cree-speaking participant.

\section{Data Collection}

The primary data collection methods were seven talking circles and five semi-structured interviews, which were transcribed verbatim. Recruitment of all participants was completed by our Cree research team member, who built relationships of trust with each one. Talking circles and interviews were conducted between March and September, 2009 and included a total of seventy-five consenting participants. A semi-structured approach was employed in both the talking circles and the individual interviews.

The talking circles included a range of participants: traditional elders, older women who were midwives or knew midwives in their communities, daughters who were proficient in maternal and infant cultural knowledge, community medicinal herbalists with experiential knowledge, and community ceremonialists. These community talking circles also included young women interested in discussing Aboriginal birthing practices, mother and infant care, and ideas about future health services within and outside their communities. The talking 
circles were approximately two hours long, and enabled in-depth discussion regarding Aboriginal maternal and infant topics. Proceedings of the talking circles were recorded simultaneously on paper, chart paper, and audiotape (with the consent of participants) by different research team members. In addition, a research team member in the group took notes that assessed interactions and kept track of salient points raised. The talking circle process was launched with a showing of a short video of a respected Cree elder whose traditions and life story as a mother and knowledge keeper of medicinal plants provided a comfortable and safe starting point for discussion. Structuring the talking circle in this way facilitated active participant engagement and exploration of emerging threads as the talking circle proceeded.

The semi-structured interviews were conducted with four respected traditional elders, who have extensive knowledge of cultural practices and understanding of current needs in their communities. The fifth interview was conducted with a young woman who had significant insights based on her own experiences of pregnancy, childbirth, and parenting a newborn. All interviewees shared at length knowledge related to cultural healing and well-being for women during pregnancy and childbirth. Interviews were approximately two hours in length and conducted by the Cree research team member. One interview and parts of two others were conducted in the Cree language, and, with the assistance of an interpreter, were simultaneously translated into English. These interpreted interviews were audiotaped and supplemented with note-taking.

The research questions were developed primarily by the Cree research team member, with input from elders and cultural experts associated with the project on how to be culturally sensitive and inclusive. Customary protocols were offered to the elders when an interview was requested. Additional input in the form of guidance on the appropriate organization of the talking circles was also provided. This guidance included instructions regarding local customs and protocols, such as how to begin the talking circles appropriately with prayer and smudging.

\section{Data Analysis}

Exploration of the complexity of Cree and Stoney women's experiences of pregnancy, childbirth, and perinatal health practices, inclusive of cultural contextual views, in each talking circle and interview was facilitated by three conversational sessions among four of the research team members after each member had independently read over the transcripts. Stories told by the participants were appreciated in their entirety during the analysis and thus coded in their entirety in an effort to respect the Aboriginal form of transferring knowledge through storytelling. This process enabled us better to identify the underlying assumptions and cultural orientations that influenced interaction between participants and healthcare providers. All data were managed manually, using data management techniques to code, organize, rearrange, and logically select components of transcript text. Several cycles of discussion and review by the research team ensured that the findings and associated conclusions were as representative as possible. 


\section{Results Part 1: The Childbirth Experience}

Five main themes emerged from the participants' descriptions and discussion of their personal and community experiences of childbirth: the exclusion of the elders, the move of childbirth away from the community, the traditional teachings related to pregnancy and childbirth, the centrality of spirituality, and the limited sense of cultural safety in the modern Western healthcare system. We explore these themes using the words of the participants.

\section{Excluding the Traditional Elders}

The experiences and stories shared by study participants stressed the break in the transmission of traditional knowledge regarding the significance and care of pregnancy and childbirth from one generation to the next. This rupture was attributed to colonialization and the subsequent sociopolitical forces impacting Aboriginal communities, including the exclusion of elders from significant community leadership roles. Women described historically changing perceptions and experiences of childbirth and maternal and infant health; previously a sacred responsibility of grandmothers and mothers, childbirth had been appropriated by the state and the Western-based medical system.

Many of the women recounted memories of traditional midwife experiences, celebration-of-life events from puberty through pregnancy and childbirth, and the reproduction of matriarchal ways of caring for and managing pregnancy and childbirth. Until the 1950s and early 1960s, all First Nations births in the Edmonton area took place within First Nations communities. One woman recalled that her mother and aunt were midwives and together they had delivered 84 babies in the community. Several others told stories of their grandmothers and mothers who were midwives trained by their mother and grandmothers.

My mother delivered babies-she was a midwife. They were all fine. One time she woke me at two [in the morning] and said Agnes was going to have her baby. This was my first experience of going with my mother to see a birth-I was five or six years old. My grandmother lived in [one of the First Nations communities in northern Alberta]. The people would come and get her and Moshom [mother] would help her ... We went many times. (Elder Participant)

Throughout pregnancy and during childbirth, women received extensive teachings and support from elders and other community members, particularly their older female relatives. The attention from older people in the community enhanced the mother's selfconfidence and sense of empowerment, because these relationships constituted a powerful and enduring connection which extended to her unborn child.

Traditionally, before childbirth, women cleaned the house. Then all women in the family go with the woman to give birth, and support her throughout the delivery. When my mother had me, I was delivered by my grandmother-born in the bush-a dream of my grandmother brought me into the world-this is not like when we went to have babies at the hospital. (Elder Participant) 
The shift of births to hospitals in the mid-twentieth century broke the historic transfer of this knowledge and these ways of caring from the elders to the younger generations. This contributed to a perception among participants that they had lost some of their collective culture and a sense of belonging:

Having your baby not at home-there's something missing-maybe caring from your mother and grandmother. You're isolated in a hospital room with doctors and so on. At home, the pain is not as heavy and it's easier to have babies at home. (Elder Participant)

Many participants lamented that their mothers and grandmothers did not teach them the art of midwifery:

My mother was a midwife although she didn't teach me-I would like to take the training to be a midwife. (Circle Participant)

While the medicalization and hospitalization of childbirth for Aboriginal women began in the mid-twentieth century (Lalonde et al. 2009), the disruption of the transmission of knowledge from generation to generation began fifty years earlier with colonization, the imposition of treaties and Christianity, and particularly the establishment of the residential school system in the late nineteenth and early twentieth centuries. Residential schools imposed religious beliefs and Western values that negatively impacted views of sexuality and teachings regarding reproductive health.

I went to residential school for three years-from age 13 to 16 . Our mother had died. At 13 I was playing with dolls and then I [went to residential school and] was taught that everything was a sin. That stayed with me my whole life. You refer to everything in life that way-you never know if it's a sin or not. Our bodies should be natural, but it doesn't happen when you have different ideas about sexuality. Childbirth is something that happened every year because birth control was a sin ... If I had been taught the facts of life instead of being taught that parts of my body were sinful, [it would have been different]. It's a shame to live like this. It makes a difference-you don't see things right-you don't ask because you don't want to sound dumb. My mother had died when I was 13-I couldn't go to an aunt or a friend. It would have been nice to have someone to share with. (Elder Participant)

Thus, not only did the residential school system interrupt the transfer of knowledge between generations, it also instilled a different view of sexuality and reproduction. The new perception was that sexuality was sinful and therefore shameful; the traditional teaching that sexuality and reproduction were to be celebrated as an integral part of life and community was lost:

These people in the school were so mean. I used to wonder why. My grandmother taught me that God was loving-in school these people were half-killing us. I was so confused when I got out-I was ashamed of being an Indian and therefore I left my grandmother's teachings. She was upset by it. The white man made you hate yourself. It took me a while to get back to those teachings. (Elder Participant) 
Because of lack of sex education, many women do not know what was happeningthey are very embarrassed, because parents didn't explain to them-because parents grew up in residential schools with shame about sex. (Circle Participant)

Today the exclusion of the elders and their guidance continues to be pervasive as young pregnant women frequently struggle without extended family and community teaching to guide them. This is troubling to elders and grandparents:

Grandparents are hurting; as we watch, our grandchildren are suffering because our children are not looking after them. (Circle Participant)

Participants discussed the loss of social support for pregnant women as elders have been excluded from the care of pregnancy and childbirth.

Our elders say when a woman is pregnant it is a very precious and special time, [and] therefore she must be treated with tenderness, love and care. A lot of this has been lost, especially in Western culture. Young women who are pregnant without a husband are judged and blamed in the Western culture and now in ours-we need to turn that around. (Elder Participant)

\section{Moving Childbirth out of the Community}

Participants described the removal of childbirth from the family and community, and its transplantation into a Western, clinical and medical setting over the course of the last two or three generations. In mainstream society, the medicalization of pregnancy and childbirththat these were increasingly managed and overseen by medical professionals, involved a high degree of technological medical intervention, and were seen as interruptions to the state of health-had been occurring since the late nineteenth century, and the practice was well-established in mainstream healthcare practice in Canada by 1920. Another contributing factor was the loss of or disregard for traditional childbirth knowledge and practices in Aboriginal communities as a result of the century-long process of colonization and cultural extermination, particularly through mechanisms such as the residential school system. In addition, by the 1980s it was government policy that all First Nations women living on rural and remote reserves be evacuated from their communities near the ends of their pregnancies to deliver in a hospital, attended by a physician, as these services were not available on-reserve. The "evacuation policy" applied to all Aboriginal women from rural and remote communities south of the 60th parallel (Lawford and Giles 2012, 331). Thus, among the participants in this study, all those over about 70 years of age-that is, those born before 1950 - were born at home with midwives. Since the 1970s, essentially all births have been in urban hospitals.

This geographic move contributed to changes in the way childbirth was viewedas a medical event, instead of part of the lifecycle of the family and community. The hospitalization of laboring mothers spatially and geographically separated pregnant and birthing women from their families and communities: 
I was born on reserve. I was at the hospital by myself for my first birth. I have two daughters and two sons, and all of them have had children. I was there for all the births. I think it's important and it is scary: I pace and pray. (Circle Participant)

I was 18 , I had no knowledge; no prenatal care. I went to the hospital and lost a lot of blood. I didn't have contact with the baby; I was too weak. I was there for two weeks; there was no one in the room, just myself. There was only one nurse for everyone. Travelling was too difficult-we had no car, just a team of horses. Men weren't allowed in the birthing room. All I remember is every time my mum would get pregnant she'd go away and come back with a baby. (Circle Participant)

I see it [pregnancy] drifting further from natural birth in my community, family and broader community. Pregnancy is not a priority or something to look forward to anymore. It is a burden now. Before, women gathered around a pregnant woman and praised, supported her pregnancy. Premature babies are starting to increase due to lack of knowledge and support as before. (Elder Participant)

For the Aboriginal women in the study, the physical place a birth occurs is important to the identity and well-being of the infant. Participants reported various ways in which the place and circumstances of birth have an influence of the life of each person.

I was born on [the] reserve-a midwife delivered me. When I was being born, they had everything prepared-it was a good feeling-[I] had a loving bond with my mother from birth and she was only 16 years old when I was born. (Circle Participant)

I was born in a tent. When I feel sick now, I like to go into a tent-it's very comforting. (Elder Participant)

According to the Elders, the separation of childbirth from the geographic and cultural community, from the woman's home community, is traumatic, and this trauma changed the way childbirth was experienced by laboring mothers. In addition to the isolation mothers felt, they also reported greater physical pain when birthing in the hospital as compared to at home or in their community.

[In the community] I didn't have to use drugs because the pain was milder. This had to do with the love these old women had and rubbing your stomach and so on. They could take the pain away-it was a big difference. In hospital you get needles, strapped down, and drugs. (Elder Participant)

\section{Recalling the Traditional Teachings about Childbirth}

Participants highlighted the way in which traditional teachings related to pregnancy and childbirth were marginalized as part of the process of colonization and assimilation that occurred over the last century. While much of this traditional knowledge has been lost, 
pockets of it still exist in the community, particularly among traditional elders, that provide evidence of the former richness and depth of this wisdom. The traditional knowledge that some elders share in their community gatherings is sometimes called "natural law," and includes such practices as the use of "cradle boards" or "moss bags," spiritual care of the baby's umbilical cord, and "amulet or medicine pouch" teachings. Participants perceived that much of the culture-specific knowledge related to herbal medicines, ceremonies, teachings, cultural practices, and songs has been forgotten. This loss is critical since this knowledge is viewed as an essential source of health and healing without which Aboriginal people cannot restore physical, mental, spiritual and emotional balance.

We lost our stories and we need them to keep balanced and a good mental state. We need to treasure our stories and natural laws. We need to respect the things Creator has given to us. Women are life givers; therefore they need to care for themselves. (Elder Participant)

The teaching as I was taught is that we're from the earth. The earth has everything here we need to heal. It's up to us to try to pray for those teachings to come to us because if you don't have access to them, then they can't help you. (Elder Participant)

Some of the teachings regarding self-care during pregnancy were gathered in the talking circles. There are taboos and restrictions about food, drinks, activity, and dress code during pregnancy. Participants recalled the following teachings from the elders regarding pregnancy self-care, for example:

Be careful of what you eat ... When you drink fluids, do not drink too hot or too cold, and stay away from salt because you will burn the blood, you will burn the baby's blood. You know how these young girls like to eat potato chips. You got to let them know to stay away from potato chips because that crunching noise hurts the baby's ears also. You will scare your baby when you have that crunching noise inside of you. (Elder Participant)

Pregnant women are not supposed to lift their arms above their heads, because the cord can get wrapped around the baby's neck. They are supposed to use a stepladder if they need to reach up high. (Elder Participant)

A Cree Elder explains how parturition was traditionally managed in the community:

When a woman begins her labour pains, there we sit with her: the grandmother, mother and the one other who helps her, a helper is there. Prayers are to be said and the mother must not be frightened and the mother must be encouraged not to cry out. The old way, we put a bar from your knees to your shoulders. That bar is about that high [ 5 feet] and we put a soft towel on it to pull on. You squat giving birth and have a clean blanket for the baby below the mother. We do not lay [sic] on our back, that's for Western birthing. It's hard on the back muscles and you could tear your womb. The old way is best. (Circle Participant) 
In a traditional delivery, there was straw and a rail that the woman could hold on to and crouch-called a "delivery stall" - sometimes they had a gag so [as] not to scream - the older girls had to watch. (Circle Participant)

Participants described what they recalled of the traditional practices related to the immediate care of the newborn and the mother, including the traditional practices related to the afterbirth.

Babies after [they are] born, we clean [them], wash [them] well and put them in soft blankets and talk to them. For woman's afterbirth to come out, make a downward motion on her stomach and the afterbirth will come out. The helper will use warm water to clean [the] mother and put that water outside in a clean place. The husband has to wait outside, [he] must not be allowed in same room. That is our way. He might become fearful. He can take the afterbirth away and bury it in a clean place. (Elder Participant)

\section{Connecting Spirituality with Childbirth}

For many Aboriginal people, the emotional, mental, and spiritual dimensions of healing and well-being are as important as the physical dimension. Many participants in the study expressed a desire to return to a holistic understanding of health by re-situating pregnancy, childbirth, and mothering within a traditional spiritual and cultural framework. Aboriginal women in the communities in this study practiced a variety of ceremonial activities, including rites of passage to mark progress along the life path and important social and physical milestones. Ceremonies existed to celebrate puberty, pregnancy, childbirth, and the passing of the placenta. These ceremonies reinforced spiritual and cultural understandings of these events and fostered belonging and social harmony within the community.

The elders would also conduct various prayers throughout pregnancy and after childbirth. They believe that praying to the Morning Star throughout pregnancy brings blessings for the baby, as explained by the quote from the elder in the introduction to this paper. In addition, several sacred ceremonies were recounted by participants as a critical part of setting the newborn on a good path for its life, including ceremonies to welcome the baby into the family and community, to name the baby, to cleanse the baby, and to return the placenta and/or umbilical cord to Mother Earth. Participants described rituals for cutting the umbilical cord and burying it in a significant place, because they believed that this will determine the child's fate. If it is buried in the woods, for example, it is believed that the child will grow to become a good hunter. The burial of the umbilical cord also provides a sense of belonging to that child.

We used to keep the umbilical cord-[this] teaches the child to stay in one placeand to put it near where the skills are, so that the child will have those skills. (Circle Participant)

If the umbilical cord is lost-then in life, the child is lost and wandering. (Circle Participant) 
Infants were traditionally brought up as members of the community, participating in all aspects of the culture. Participants described ways in which the elders and extended family introduced the child into their cultural community.

When the babies are born, they take them in the sweat [lodge] as newborns. The women don't [go into the] sweat until their flow stops. (Circle Participant)

It is the responsibility of the grandmother or grandfather to give grandchildren "Indian names"-even though some people make fun of this. This helps keep them on the right road. (Circle Participant)

\section{Limited Cultural Safety in the Healthcare System}

The removal of childbirth from the community to the hospital resulted in poorer childbirth experiences and health outcomes for the majority of Aboriginal women participants in this study, echoing the negative impact of this shift in Aboriginal communities throughout Canada (Lalonde, Butt, and Bucio 2009; Allan and Smylie 2015). It is not known whether this shift has actually resulted in outcomes that are worse than they were before the removal took place; however it is known that health outcomes continue to remain worse for First Nations compared to non-First Nations. Smylie and Adomako (2009), for example, indicate that among First Nations people with status, infant mortality is almost twice the rate of the general Canadian population (mortality rates for non-status First Nations were not available), and among First Nations, both low birth-weight rates and high birth-weight rates are higher than among the general Canadian population.

In their stories about their experiences of hospital births, the participants revealed an absence of understanding of what was going on, a lack of access to information to make informed decisions, perceptions of discrimination, and perceived institutional and institutionalized bias. Most of the participants described hospital birth experiences in which they had little preparation for what to expect and little understanding of what was being done to them. They did not feel that healthcare providers readily provided information or explanations. The following story illustrates this lack of understanding and the bewilderment, grief, and anger that can accompany it.

I lost a little girl at twenty weeks at [a city hospital]. The baby was born alive-she was the size of my hand. She was kept on my chest until she passed away. They didn't keep her in an incubator. Maybe at [another hospital], they would have put her in an incubator or something. (Circle Participant)

Another woman's story highlights not only the challenges of getting to the hospital, but also the misunderstandings that can happen with healthcare providers.

I was 21 when I had my first child; I got to [a city hospital]. There was a blizzard outside and I was at my sister's place. It was cold-November tenth. We tried to start the truck and the tractor and neither started. The first set of car lights come up the hill-you are off to the hospital. I got in the elevator, They wouldn't answer 
any questions. I went upstairs - they never told you where, they just took you. The nurse said: "She's not helping herself." I asked "What are you talking about?" The nurse said "Did anyone explain birth to you?" I guess I wasn't pushing, I didn't know. No one said anything or told you anything, you just went. (Circle Participant)

Many participants felt that healthcare providers did not have an appreciation of Aboriginal sensibilities and sensitivities regarding their bodies, particularly in relation to personal modesty and safety. Traditional Aboriginal culture was highly discreet and respectful about nudity; only female relatives and elders would care for the woman in labour. The prevalence of historic and contemporary physical and sexual abuse within Aboriginal communities also heightens perceptions of violation when individuals are not treated with respect and gentleness.

I believe the most difficult time is the birthing, strangers helping and some are men. I see my sister who had only to deal with men doctors. [Giving birth should be] a sacred time. Young mothers aren't able to deal with these things; there is a no [cultural] place, space for it. No choices for birthing today for women. Pregnancy by force, rape, aggression - that trauma at birth by a stranger [doctor] again, is not healthy thing. (Elder Participant)

At the hospital—there were curtains and a soap dish. They said, "Take your panties off." The nurse was going to shave me but didn't explain it. I had to ask all the questions. I was shocked to be asked to take my panties off. (Circle Participant)

Aboriginal women's responses to pain were also mentioned as an area of misunderstanding. Several participants commented that stoic behaviour toward pain in childbirth was valued in traditional culture. "Suffering in silence" was considered a virtue. This silence was often misunderstood by healthcare providers, who then missed important cues for maternal care and pain management.

While some participants described positive interactions with hospital healthcare providers, many felt they experienced discrimination and harsh treatment in mainstream medical facilities. They noted that racism, common stereotypes, and general lack of awareness about Aboriginal people seemed to influence attitudes and treatment in the hospital:

I had my first baby at [a city hospital]. They were very prejudiced. I was treated harshly. One lady said "My baby's coming" - they said "Oh you're just drunk," and the baby fell right there. (Circle Participant)

Often, official hospital policies did not accommodate the cultural and social needs of Aboriginal women during labour and delivery.

I had the first six [babies] at [one city hospital], and the seventh at [another city hospital] because she was so early. With the doula, I wanted a home birth, but the hospital didn't encourage the family to be around. We had fifteen people there-the staff was quite irritated. (Circle Participant) 
Each of my girls had a doula-but they had to cancel because we were only allowed two people in with the mother. They shouldn't have interns etc. in there [the birthing room]. We need to have grandmothers and sisters and so on in there. We need key people in the extended family. (Circle Participant)

\section{Results Part 2: Recommendations}

The elders and participants in the talking circles were unanimous in their desire for a healthcare system that combined the best of Western medicine and the holistic, familycentred approach of traditional Aboriginal communities. The participants did not want to forego the benefits of Western medicine; rather, they wanted a respectful, harmonized integration of Aboriginal ways of knowing and doing with those of the Western healthcare system.

We need Western medicine for complications, but [we need] our families and communities for support and health. (Elder Participant)

It is in seeking a sociocultural analysis approach that a discovery of indigenous perspectives, medicine, and therapies may be understood as an indigenous health system, rendering recognition, respect, and understanding of their knowledge and resources to improve health and well-being (PAHO 2011). Thus, rather than one system being dominant or negating the other, a harmonization approach would respect the integrity of each system of medicine, and value and access both systems as appropriate.

According to the study participants, four key elements are needed to create an integrated healthcare service: elders and family; cultural supports and practices; Aboriginal "doulas" or midwives; and culturally safe healthcare providers in hospitals.

\section{Aboriginal Elders and Family Support}

The first and central element of the participant recommendations was the need to bring elders, grandmothers, and other key female relatives back into the circle surrounding pregnancy in the community and birthing women in hospitals. The elders and grandmothers are essential to providing traditional wisdom and guidance, and well as spiritual grounding for women in childbirth:

It is important to us that we bring elders to our gatherings to share and bring forward teachings in our language of the traditional practices of our ancestors to continue the traditional ways of our ancestors. We believe it is highly important to our health and well-being into the future, for the next seven generations. (Elder Participant)

Old people are being put aside. Now they are trying to bring them back to teach [others] how to live and respect yourself. They [the elders] are just about all gone, yet they are not included. I went to a meeting here a few years back. There were a few elders talking about how to get the elders involved. The elders were busy playing bingo-no one asking them for help—there is nothing for them to do. We 
need to give them tobacco and ask them ... We need to involve elders-that's the only way-they don't feel wanted. (Elder Participant)

It was also very clear that study participants felt that the presence of other key female relatives and valued members of the extended family provided needed additional support and care. While many clinical settings are not designed for extended family accompaniment in labour and delivery rooms, this appears to be an important consideration for many of the participants:

Medical facilities need to have a place where grandmothers can be. You could have mother and aunties with her [the woman in labour] for a while-in one hospital all the staff left for a while so that they could be alone with the mom. It's important that family can be in the hospital with the birthing mom. (Circle Participant)

As women, we are strong, but sometimes we need support-I need support when having a hard time; when we have done all we can do, and we need help, we need to reach out. (Circle Participant)

\section{Cultural Supports and Practices}

The second element in such a program, which is closely linked with the reinvolvement of elders, is to revalue traditional teachings and practices that have been marginalized, and have these available to those who request them, as a means of enhancing the meaning and success of the pregnancy and childbirth experience.

The only way I see it working today is to have knowledge of natural law. That is the missing link for our people. That was taken away from us in 1885 with the signing of the treaties. That law was made that we could not do any more ceremonies and natural law. We need to understand that that's where we get our mind from, healthy mind, healthy heart, healthy family, and healthy child-rearing. That's what I know is the missing link ... We must teach women this. (Elder Participant)

Now there is a trend for young people learning the old ways ... If we could incorporate Aboriginal women who are midwives into the Western world, in the prenatal classes maybe; if we could have that and support our elders in helping and teaching our women and our young men. [It's] important to share our stories and they need to get to know themselves - then they can pass that security to the baby. (Elder Participant)

Participants viewed it as a positive first step that some of the local hospitals now have "Aboriginal Cultural Helpers" hired into positions to offer "Welcome Baby" ceremonies and smudging for families who request this. Several hospitals also have policies that enable parents to take the placenta and/or umbilical cord home for ceremonial purposes. Policies regarding the number of family members permitted to support a birthing mother or visit a hospitalized newborn need to be reviewed to allow for a circle of support to be present 
for the labouring mother and her baby. The policies and facilities that might enable these cultural supports and practices need to be enhanced, according to study participants.

Within the communities, there are already efforts underway to recover some of the cultural and spiritual practices which build physical, sexual, and reproductive health, particularly opportunities for female elders to teach young women throughout the lifecycle, as is described in the two cases below:

Teaching girls about menstruation and sexual health is a four-day "rites of passage" it shapes adult life, it's time with four elders. This is still done with many girls today. (Circle Participant)

My dad's mother was a midwife in our community and she taught my mother all that she knew. I've always been concerned about my granddaughters and daughters. My sister and I began a circle to teach the girls. We started with a feast. All the females bring a gift for the girl who has her time [first menses] and we all tell her that it is special. (Elder Participant)

\section{Aboriginal "Doulas" and Midwives}

Trained Aboriginal community-based maternal-infant health specialists, in a doula role, or even as midwives, would be the third component of an integrated program. This person located in, and preferable a resident of, the First Nations community would accompany the pregnant women through the entire process, and provide prenatal teaching, connections, support, and a liaison role with healthcare professionals in the mainstream system as needed. It was recognized that many young women are already seeking support from doulas before birth (e.g. prenatal classes) and after birth (e.g. training on breastfeeding), in addition to during the intrapartum period. Participants in this study envisioned this accompaniment as providing numerous benefits:

A doula could help monitor the pregnancy so women do not end up delivering alone. We could have people in the community who have experiential knowledge that could do this role-they just need the confidence and certification to get into the classroom. Often the elders are tired, but maybe the younger grandmothers could accompany pregnant women. (Elder Participant)

\section{Culturally Safe Healthcare Providers}

The fourth critical component in a program responsive to Aboriginal women's needs is to have healthcare providers who are culturally competent and act in a context of cultural safety. Participants felt that, at a minimum, this approach prevents discrimination and prejudice, and at best, it can facilitate a mutual respect and caring in the realm of health service delivery. It is only with the personal expertise to negotiate a relationship based on authentic mutual respect, which is also reciprocal and constantly interchanged, that knowledge derived from understanding Aboriginal maternal/infant/family/community 
experiences emerges. Participant experiences revealed that through respectful relationships and communication, Aboriginal people and healthcare providers can identify and implement authentic, culturally relevant, and competent healthcare practices that meet the needs of clients in a holistic manner. According to one elder, this type of reciprocal learning can improve health outcomes for future generations.

It is important to us that we bring them [healthcare providers] to our gatherings to share and bring forward teachings in our language of the traditional practices of our ancestors to continue the traditional ways of our ancestors. We believe it is highly important to our health and well-being into the future, for the next seven generations. (Elder Participant)

\section{Conclusion}

This study contributes to the emerging area of inquiry and dialogue exploring how Aboriginal cultures and traditions can have a greater role in contemporary contexts of childbirth. The Cree and Stoney women and elders who participated in this study shared their experiences of loss and separation as pregnancy care and childbirth moved out of the community and into hospitals over the last two generations. The removal of childbirth from the community to the hospital was not only a geographic relocation, it was also a sociocultural upheaval as the childbirth experience was disconnected from elders, family and community, traditional teachings, and spiritual meaning. Conversely, participants' hospital experiences, while benefiting from modern Western medicine, have tended to include a limited sense of cultural safety and grounding.

Both community members and traditional elders highlighted the urgent need to restore culturally-based community support and health perspectives to the reproductive experience. Participants suggested that an Aboriginal community health framework operating in parallel with the Western biomedical system could create the conditions for healthier Aboriginal mothers and newborns. Four key components for this harmonized healthcare model were identified: reintegration of grandmothers and elders into care; availability of culturally-based health teachings and practices; trained Aboriginal community-based maternal-infant health doulas and/or midwives; and culturally competent healthcare providers. Implementation of this type of harmonized health model for maternal-infant healthcare in all Aboriginal communities-remote, rural, suburban, and urban-may be the key to finally closing the gap between Aboriginal and non-Aboriginal health status in Canada, and restoring the "blessings of the Morning Star." 


\section{Bibliography}

Adelson, N. 2005. "The Embodiment of Inequity: Health Disparities in Aboriginal Canada." Canadian Journal of Public Health 96: S45-S61.

Allan, B., and J. Smylie. 2015. First Peoples, Second Class Treatment: The Role of Racism in the Health and Well-being of Indigenous Peoples in Canada. Toronto: The Wellesley Institute.

Birch, J., L. Ruttan, T. Muth, and L. Baydala. 2009. "Culturally Competent Care for Aboriginal Women: A Case for Culturally Competent Care for Aboriginal Women Giving Birth in Hospital Settings.” Journal of Aboriginal Health 4(2): 24-34.

Chandler, M. J., and C. Lalonde. 1998. "Cultural Continuity as a Hedge against Suicide in Canada's First Nations." Transcultural Psychiatry 35(2): 191-219.

Chandler, M.J., and C. Lalonde. 2008. "Cultural Continuity as a Moderator of Suicide Risk among Canada's First Nations.” In Healing Traditions: The Mental Health of Aboriginal Peoples in Canada, edited by L. J. Kirmayer and G.G. Valaskakis, 221248. Vancouver: UBC Press.

Claydon, J. E., Mitton, C., Sankaran, K., Lee, S. K., and the Canadian Neonatal Network. 2007. "Ethnic Differences in Maternal Risk Factors and Infant Outcomes in Canadian High-Risk Births.” Perinatology 9(1): 1-8.

Couchie, C., and S. Sanderson. 2007. "A Report on Best Practices for Returning Birth to Rural and Remote Aboriginal Communities." Journal of Obstetrics and Gynecology Canada 188: 250-4.

Foster, C. H. 2006. "What Nurses Should Know When Working in Aboriginal Communities." Canadian Nurse 102(4): 28-31.

Greenwood, M. 2005. "Children as Citizens of First Nations: Linking Indigenous Health to Early Childhood Development." Pediatric Child Health 10(9): 553-5.

Inter-Agency Support Group on Indigenous Peoples' Issues. 2014. The Health of Indigenous Peoples. Thematic paper towards the preparation of the 2014 World Conference on Indigenous Peoples.

Kirmayer, L., and G. G. Valaskakis. 2009. Healing Traditions: The Mental Health of Aboriginal Peoples in Canada. Vancouver: UBC Press. 
Lalonde, A. B., C. Butt, and A. Bucio. 2009. "Maternal Health in Canadian Aboriginal Communities: Challenges and Opportunities." Journal of Obstetricians and Gynecologists of Canada 31(10): 956-62.

Lawford, K., and A. Giles. 2012. "An Analysis of the Evacuation Policy for Pregnant First Nations Women in Canada." AlterNative: An International Journal of Indigenous Peoples 8(3): 329-42.

Lang, C., M. E. Macdonald, F. Carnevale, M. J. Lévesque, and A. Decoursay. 2010. "Kadiminekak Kiwabigonem: Barriers and Facilitators to Fostering Community Involvement in a Prenatal Program in an Algonquin Community." Pimatisiwin: A Journal of Aboriginal and Indigenous Community Health 8(1): 55-81.

Luo, Z.-C., R. Wilkins, M. Heaman, P. Martens, J. Smylie, L. Hart, F. Simonet, S. Wassimi, Y. Wu, and W.D. Fraser. 2010. "Birth Outcomes and Infant Mortality by the Degree of Rural Isolation among First Nations and Non-First Nations in Manitoba, Canada." The Journal of Rural Health 26: 175-181.

Martin Hill, D. 2009. “Traditional Medicine and Restoration of Wellness Strategies.” Journal of Aboriginal Health 5(1): 26-42.

Minkler, M., and N. Wallerstein. 2008. Community-Based Participatory Research for Health: from Process to Outcomes. San Francisco: Jossey-Bass.

Moffit, P. 2004. “Colonization: A Health Determinant for Pregnant Dogrib Women.” Transcultural Nursing 13: 323-30.

National Aboriginal Health Organization (NAHO). 2004. Maternal/Infant Health and Aboriginal Maternal/Infant Health in Canada. Ottawa: NAHO.

Olson, R., K. Bebee, J. Benedict, E. Blais, E. Harney, and S. Wolfe. 2012. "Introduction: Prioritizing Indigenous Maternal and Infant Health.” Pímatísíwín: A Journal of Aboriginal and Indigenous Community Health 10(3): 267-70.

PAHO. 2011. Harmonization of Indigenous and Conventional Health Systems in the Americas. Washington DC: Pan-American Health Organization (PAHO).

Reading, J., A. Kmetic, and V. Gideon. 2007. First Nations Wholistic Health Policy and Planning Model: Discussion Paper for the World Health Organization Commission on Social Determinants of Health. Ottawa: Assembly of First Nations. 
Sandelowski, M. 2000. "Focus on Research Methods: Whatever Happened to Qualitative Description?" Research in Nursing \& Health 23: 334-40.

Skye, A. 2010. "Aboriginal Midwifery: A Model for Change." Journal of Aboriginal Health 6(1): 28-37.

Smylie, J., \& Adomako, P. 2009. Indigenous children's health report: Health assessment in action. Toronto: Centre for Research on Inner City Health, The Keenan Research Centre in the Li Ka Shing Knowledge Institute, St. Michael's Hospital. Retrieved from www.stmichaelshospital.com/crich/indigenous_childrens_health_report.php

Smylie, J., D. Fell, and A. Ohlsson. 2010. "A Review of Aboriginal Infant Mortality Rates in Canada: Striking and Persistent Aboriginal/Non-Aboriginal Inequities." Canadian Journal of Public Health 101(2): 143-8.

Society of Obstetricians and Gynecologists of Canada (SOGC) 2010. "Returning Birth to Aboriginal, Rural, and Remote Communities." SOGC Policy Statement 251: 1186-8.

Whitty-Rogers, J. 2006. "Mi'kmaq Women's Childbirth Experiences: Summary of the Literature." Pimatísiwin: A Journal of Aboriginal and Indigenous Community Health 4(1): 67-93.

Wright, A. L. 2015. "Role of the Nurse in Returning Birth to the North." Rural and Remote Health 15:3109. Online 2015 www.rrh.org.au http://rrh.org.au/articles/ showarticlenew.asp?ArticleID=3109. 